OAl-PMH: http://www.indteca.com/ojs/index.php/Revista Scientific/oai

Artículo Original / Original Article

\title{
Evaluación de los Aprendizajes un Tema Indispensable desde las TIC: Reflexionando en un Contexto Global
}

\author{
Autora: Elsy Jackelin Bracho Hernández \\ Universidad Pedagógica Experimental Libertador, UPEL \\ docelsybracho@gmail.com \\ Puerto Cabello, Venezuela \\ https://orcid.org/0000-0001-7056-0298
}

\section{Resumen}

Este estudio se centra en comprender la valoración del aprendizaje en los programas universitarios para lo cual se propone indagar sobre la teoría del aprendizaje del conectivismo de Siemens (2005), el principal propósito de este artículo es enumerar algunos elementos que proporcionen una articulación con esta teoría, también señalar algunos componentes resaltantes en relación a esta teoría considerando la modalidad de estudio de los programas universitarios y por ultimo proponer desde una concepción amplia un modelo de evaluación innovadora con aplicación en las Tecnologías de la Información y la Comunicación (TIC), en base a lo expuesto este trabajo se apoya en una investigación cualitativa, sustentada por Monje (2011), el método a seguir para la comprensión del mismo es el fenomenológico hermenéutico de Heidegger (1989). Como técnica de investigación se plantea el análisis documental. Dentro de las conclusiones se aprecia que la educación a distancia es un camino que apenas está iniciando en algunas universidades, porque algunos profesores tradicionalistas temen al cambio, también se aprecia que los profesores deben trabajar más en las murallas que se trazan, sin embargo, es una experiencia gratificante para los participantes, además la valoración del aprendizaje permite conectar teoría y práctica a través de una evaluación formativa de retroalimentación.

Palabras clave: evaluación; aprendizaje; tic.

Código de clasificación internacional: 5802.04 - Niveles y temas de educación.

\begin{abstract}
Cómo citar este artículo:
Bracho, E. (2021). Evaluación de los Aprendizajes un Tema Indispensable desde las TIC: Reflexionando en un Contexto Global. Revista Scientific, 6(21), 163-179, e-ISSN: 2542-2987. Recuperado de: https://doi.org/10.29394/Scientific.issn.2542-2987.2021.6.21.8.163-179
\end{abstract}

Fecha de Recepción: 08-03-2021
Fecha de Aceptación: 03-07-2021
Fecha de Publicación: 05-08-2021 
OAl-PMH: http://www.indteca.com/ojs/index.php/Revista Scientific/oai

Artículo Original / Original Article

\title{
Assessment of Learning an Indispensable Topic from ICT: Reflecting in a Global Context
}

\begin{abstract}
This study focuses on understanding the assessment of learning in university programs for which it is proposed to inquire about the learning theory of connectivism by Siemens (2005), the main purpose of this article is to list some elements that provide an articulation with this theory, also to point out some outstanding components in relation to this theory considering the study modality of university programs and finally to propose from a broad conception an innovative evaluation model with application in ICT, based on the above, this work is supported by a qualitative research, supported by Monje (2011), the method to follow to understand it is the phenomenological hermeneutic of Heidegger (1989). As a research technique, documentary analysis is proposed. Among the conclusions, it is appreciated that distance education is a path that is just beginning in some universities, because some traditionalist teachers fear change, it is also appreciated that teachers must work more on the walls that are drawn, however it is a rewarding experience for the participants, in addition the assessment of learning allows to connect theory and practice through a formative evaluation of feedback.
\end{abstract}

Keywords: evaluation; learning; ict.

International classification code: 5802.04 - Levels and subjects of education.

How to cite this article:
Bracho, E. (2021). Assessment of Learning an Indispensable Topic from ICT: Reflecting in a
Global Context. Revista Scientific, 6(21), 163-179, e-ISSN: 2542-2987. Recovered from:
https://doi.org/10.29394/Scientific.issn.2542-2987.2021.6.21.8.163-179

Date Received:

08-03-2021
Date Acceptance:

03-07-2021
Date Publication: 05-08-2021 


\section{Introducción}

Esta investigación muestra la evaluación de los aprendizajes desde múltiple perspectiva, ya que es un fenómeno que influye en los estudiantes de acuerdo a las circunstancias que se tengan, es decir en determinados casos son generados por factores positivos o negativos, por ende de esta dependen: los aciertos y desaciertos, los logros alcanzados, las competencias adquiridas, la deserción de los participantes, falta de interés hacia el mejoramiento profesional, baja autoestima, poca calidad profesional.

La evaluación de los aprendizajes se ha venido desarrollando a partir del siglo II con la China imperial, sin embargo, es a partir del siglo XX que se considera la medición psicológica de rasgos individuales, en tal sentido se mencionan algunos conceptos de acuerdo con algunos autores con el fin de demostrar su evolución a través del tiempo. Y las implicaciones de esta, en la práctica educativa.

Este espacio para la reflexión está orientado por los siguientes propósitos: enumerar algunos elementos que proporcionen una articulación con la teoría de aprendizaje: el conectivismo de Siemens (2005a): y la valoración del aprendizaje, señalar algunos componentes resaltantes con relación a esta teoría considerando la modalidad de estudio de los programas universitarios, al proponer desde una concepción amplia un modelo de evaluación innovadora con aplicación en las TIC.

En tal sentido es necesario conocer cómo ha evolucionado la evaluación de los aprendizajes atendiendo las obras de algunos autores, exponer dentro de la valoración del aprendizaje el papel que juegan los recursos tecnológicos en la evaluación con aplicación en las TIC, para esbozar la importancia de las plataformas virtuales en los tiempos actuales, en este sentido se consideraron unos documentos de donde se tomaron algunos conceptos. En virtud de obtener datos más recientes del tema se recogen aportes de algunos artículos de donde se extraen las conclusiones para 
vislumbrar el fenómeno y reflexionar al respecto.

Este artículo fue enfocado en base al paradigma cualitativo, bajo la orientación del método fenomenológico hermenéutico, esta dualidad permitió describir los hechos e interpretarlos para obtener las reflexiones finales del mismo.

\subsection{Teoría del aprendizaje del conectivismo de Siemens (2005)}

Mediante la investigación realizada se pudo apreciar que por medio de esta teoría se puede obtener un aprendizaje, debido a la información que se encuentra en las redes, pero es necesario entender que no toda esa información es válida, es importante diferenciar cual es la que verdaderamente va a ampliar el conocimiento que ya se posee. Por otra parte, algunos factores relevantes de esta teoría implícitos en el conectivismo son:

a). Se puede aprender y adquirir conocimiento a través de los diferentes pensamientos expuestos en las redes.

b). El aprendizaje se obtiene usando conexiones entre recursos tecnológicos de información.

c). Una forma de mantener conocimiento constante es por medio de la vinculación con las redes.

d). Un perfil importante de un alumno es la destreza para hacer una vinculación entre diferentes nociones, pensamientos, percepciones.

e). Los datos disponibles en la red en el presente pueden ser alteradas por el cambio constante de opiniones de la realidad por lo tanto es relevante cuando se investiga elegir las ideas que posibiliten la ampliación de los conocimientos.

\subsection{Evaluación del aprendizaje desde la concepción de varios autores}

Una de las grandes ocupaciones que ha tenido la evaluación se ha generado de las técnicas y los instrumentos. La implementación de las 
técnicas resultado de la psicología se prestó a concluir en algún momento que se debían elaborar las pruebas bajo supervisión de expertos, formar y asesorar a los profesores para el diseño de instrumentos válidos y confiables. En atención a lo expuesto es favorable resaltar en este estudio algunos conceptos definidos por diferentes autores.

En este sentido, Gronlund (1973), citado por Villamizar (2005): menciona que "es un proceso sistemático para determinar hasta qué punto alcanzan los alumnos los objetivos de la educación" (pág. 542). Es decir, el docente una vez que haya ordenado sus registros definirá si los estudiantes lograron las metas trazadas. Asimismo, Mager (1975): establece que es un procedimiento donde se trata de describir las cualidades que se poseen en base a una determinada meta. A lo que se refiere este autor es que se establecen dos elementos importantes en la evaluación: definir los objetivos y en base a los mismos realizar el proceso para evaluar, donde de forma descriptiva se obtiene un resultado del estudiante el cual puede ser positivo o negativo.

Por su parte, De la Orden (1982), citado por Ruiz (2001): asume que es un "proceso de recogida y análisis de la información relevante para descubrir cualquier faceta de la realidad educativa y formular un juicio sobre su adecuación a un patrón o criterio previamente establecido, como base para la toma de decisiones" (pág. 195). hace referencia que antes de tomar decisiones es importante conocer lo que existe, pero antes es primordial reunir argumentos para hacer una valoración real.

Por otro lado, Cano (2008), citado por Bautista (2016): explica que es un "proceso que utiliza diversidad de instrumentos e implica a diferentes agentes, con el propósito de proporcionar información sobre la progresión en el desarrollo de la competencia y sugerir caminos de mejora" (pág. 129). Contempla la importancia de los instrumentos para evaluar y conocer sobre las capacidades y evolución del individuo con la intención de meditar en los 


\section{Artículo Original / Original Article}

avances educativos.

A continuación, se toman en consideración otros estudios en donde se aprecia la evaluación como un proceso para favorecer el aprendizaje y un perfil de un docente estratégico capaz de crear las mejores condiciones para cumplir con las expectativas de formación en los estudiantes. En referencia al tema, López y Palacios (2012), aportan que:

El panorama poco alentador de los sistemas de evaluación en las aulas universitarias, la literatura internacional especializada lleva muchos años acumulando estudios y evidencias sobre la necesidad de utilizar la evaluación como estrategia para mejorar y favorecer los aprendizajes, y no como simple certificación del éxito o fracaso (pág. 337).

Haciendo énfasis ante lo expuesto, es sin duda un acierto, se debe tener como requisito primordial, que lo básico al evaluar son las habilidades y destreza del docente en buscar las técnicas apropiadas que permitan que los estudiantes aprendan de manera independiente, el éxito radica en realizar un buen proceso para obtener una buena formación. En este sentido, Rosales (2014), concluye que el docente:

Tiene un nuevo rol en las evaluaciones, que se asemeja más al de un examinador externo y moderador, ya que debe controlar el proceso, proteger a los estudiantes de las puntuaciones injustas y establecer los criterios de referencia para la evaluación. En el campo educativo, la evaluación permite descubrir que los objetivos planteados se han cumplido o no, lo que servirá para retomar aquellos que no fue asimilado por los alumnos, reforzar los éxitos obtenidos y no incurrir en los mismos errores en el futuro, para lo cual será conveniente introducir el cambio de estrategias pedagógicas para enmendar lo insuficiente (pág. 11).

Es conveniente aportar que en muchos casos los estudiantes se sienten atropellados por los docentes y por tal razón se sienten desmotivados, pierden el interés y dejan de esforzarse al realizar sus actividades porque consideran 
que los profesores carecen de pedagogía y de ética en la mayoría de los casos y realmente se debe a que este no sigue el proceso adecuadamente, algunos puntos a considera en este aspecto son: objetivo que el estudiante debe lograr, estrategias empleadas de acuerdo a esas metas, aplicar la evaluación formativa, orientar a los estudiantes en el proceso. Acorde con esto, Púñez (2015), manifiesta que:

Es difícil lograr una cultura evaluativa, mientras el enfoque de la política educativa y evaluativa son herméticas a otro tipo de evaluación que va mucha más a un enfoque formativo. Dentro de los enfoques cognitivos y de la evaluación para el aprendizaje, el error, la motivación, y realimentación del docente son elementos y condiciones importantes para mejorar el aprendizaje de los estudiantes. Existe confusión entre examen y evaluación, pues los instrumentos, pruebas y exámenes que adquieren medición y calificación en muchos casos generan malestar, temor, ansiedad, estrés y otras conductas emocionales en el estudiante (pág. 96).

En lo que respecta al párrafo anterior, a pesar de los avances y los estudios realizados relacionados a la evaluación de los aprendizajes, seguimos estancado en un modelo de evaluación tradicionalista, muchos profesores están cerrados al cambio, es imprescindible nuevos paradigmas que estén orientados por un proceso formativo.

Por otro lado es necesario destacar que algunas personas tienen una concepción inequívoca de este proceso al punto de considerar que realizan de manera efectiva la evaluación formativa al hacer firmar al estudiante al finalizar un periodo o trimestre escolar las notas, hasta que no comprendan que se trata de una formación que le permita al estudiante hacer una retroalimentación en donde conozcan sus fortaleza y debilidades y en base a eso hacer las correcciones pertinentes, no se avanzará en este proceso. En conformidad con Pérez, Enrique, Carbó y González (2017):

Es indispensable tener una concepción integradora de la evaluación, cuestión que es considerada la esencia del 
proceso evaluativo. Esto permite disponer de un docente competente, capaz de aplicar una adecuada evaluación formativa efectiva a través de la utilización de métodos diversos, según los objetivos que se esperan de los estudiantes de acuerdo con su nivel de estudios. Una adecuada evaluación formativa asegura que el estudiante desarrolle mecanismos de autorregulación siempre dirigidos a alcanzar nuevas metas de aprendizaje (pág. 279).

\subsection{Evaluación con Aplicación en las TIC}

Este tipo de evaluación se basa en recolectar información sobre las actividades asignadas para la valoración del pensamiento alcanzado por los estudiantes, utilizando los recursos tecnológicos favorables para tal fin. Al respecto, Barberà (2016): describe que en "[...] la tecnología se resaltan tres grandes cambios que la tecnología ha aportado en el contexto de la evaluación" (pág. 5); estableciéndose de la siguiente manera: evaluación automática, evaluación de tipo enciclopédico y evaluación colaborativa.

Adicionalmente, Peñalosa (2010): propone "un modelo de aplicación del Análisis Cognitivo de Tareas en la investigación del aprendizaje en línea" (pág. 30). En este se trabajan dos campos esenciales: "[...] la evaluación y la interactividad [...]" (pág. 30); basados en diez (10) pasos o fases: "[...] evaluaciones colaborativas; modelación del dominio educativo; diseño instruccional; evaluaciones diagnósticas; evaluaciones formativas; las evaluaciones sumativas; materiales de aprendizaje; análisis de las interacciones; evaluaciones automáticas; evaluaciones elaborativas [...]" (págs. 30-32).

Las tareas en línea juegan un papel esencial en la motivación y aprendizaje de los participantes, ya que permite una interacción entre los recursos y el medio, los estudiantes, profesores y a su vez, existe una conexión pertinente entre todos los elementos expuestos, principalmente se destaca la implementación de los tipos de evaluación y métodos para desarrollar los 
temas en conjunto, todo lleva a lograr metas de aprendizajes.

\subsection{Evaluación de los aprendizajes desde la plataforma Moodle}

La plataforma Moodle es un recurso en donde la experiencia es agradable, las bondades que ofrece permiten ampliar los conocimientos y las capacidades creativas, en donde el intercambio de pensamiento y la cooperación son pieza fundamental, se evidencia a través de la retroalimentación, las ideas y tareas compartidas por los participantes.

Se puede considerar que "Moodle" es una de las favorita de los todos los que participan en ella debido a que esta posee diversidad de oportunidades para incluir en la modalidad de estudios a distancia y presenciales permitiendo a su vez hacer modificaciones de acuerdo a las clases y también al grado de complejidad de los cursos o programas, sin embargo algunos de los estudiantes solo se registran otros no son capaces de seguir el compás, por lo tanto no continúan, entonces en ese aspectos deberían ser específicos con las instrucciones.

\section{Metodología}

Este estudio se fundamenta en una investigación cualitativa, debido a que a través de esta se presentaron documentos con el propósito de interpretarlos para conocer aspectos importantes relacionados a la evaluación de la enseñanza en los programas universitarios y llegar a enumerar algunos elementos que proporcionen una articulación con la teoría del aprendizaje del conectivismo. Al respecto, Monje (2011a): expresa que la investigación cualitativa "[...] busca conceptualizar sobre la realidad con base en el comportamiento, los conocimientos, las actitudes y los valores que guían el comportamiento de las personas estudiadas" (pág. 13).

Además el estudio centró el análisis en la descripción, explicación y comprensión del fenómeno en donde se apreciaron los resultados por medio 
del método fenomenológico hermenéutico de Heidegger (1989): citado por Barbera y Inciarte (2012): ellas señalan que el propósito del mismo "es apropiarse del significado ya implícito en la experiencia vivida, mediante un proceso de pensamiento orientado por la destrucción y construcción hasta lograr interpretarlo como su verdad; esto es, revelar los fenómenos ocultos y, en particular, sus significados" (pág. 202).

Se refiere a que cada persona tiene una percepción de las cosas de acuerdo con sus vivencias y logra esos pensamientos por medio de la comprensión y el entendimiento. Como técnica se expone el análisis documental de Monje (2011b): el cual consistió en analizar la información contenida en artículos de revista para descubrir el significado que le suministran los autores a la evaluación de los aprendizajes y proponer desde una concepción amplia un modelo de evaluación innovadora con aplicación en las Tecnologías de la Información y la Comunicación (TIC).

Como instrumento se consideró el análisis de contenido cualitativo, donde se presentaron conceptos de algunos autores y artículos de revista para obtener los resultados del estudio se toman los conceptos expuestos y se reflexiona al respecto, también se consideran los contenidos señalados en la investigación sobre la TIC.

\section{Resultados y Discusión}

En atención a enumerar algunos elementos que proporcionen una relación con la teoría de aprendizaje: el conectivismo de Siemens (2005b), y la evaluación de los aprendizajes se entiende lo siguiente:

1. Evaluar es una necesidad para el poseso de enseñar y aprender, lo cual implica desarrollar competencias, alcanzar metas, lograr objetivos, mejorar el aprendizaje y desarrollar capacidades de autorregulación, en este sentido se puede aprender y adquirir conocimiento a través de los diferentes pensamientos expuestos en las redes., donde es necesario 
la destreza del alumno para vincula diferentes nociones, pensamientos, percepciones como un potencial a desarrollar entre los estudiantes, por lo tanto este proceso de aprendizaje radica en mantener conocimiento constante por medio de la vinculación con las redes o plataformas virtuales.

2. Es un proceso de valoración sistemática para lo cual se deben no solo recoger datos sino registrarlos, para luego analizarlos, esta parte tiene que ver con las estrategias y procedimientos que se utilicen, en el cual es esencial las conexiones entre medios de información y comunicación para obtener conocimiento constante. Aplicando en el aprendizaje los múltiples mecanismos que puedan ser útiles, entendiendo que este puede encontrarse en las plataformas virtuales y puede ser tan efectivo como el que se consigue de la forma tradicional.

3. Tomar decisiones luego de una emisión de juicios es fundamental, se refiere a la reflexión que se obtiene luego de un proceso sistemático sobre la valoración del estudiante, entonces en atención a la teoría los datos disponibles en la red en el presente pueden ser alterados por el cambio constante de opiniones de la realidad por lo tanto es relevante hacer una buena decisión cuando se investiga eligiendo las ideas que posibiliten la ampliación de los conocimientos.

Se considera que las relaciones expuestas se fusionan y le dan sentido a ese proceso de evaluación que en muchas ocasiones se toma a la ligera sin tomar en cuenta los diferentes factores implícitos para llegar a una formación en el que los estudiantes realmente construyan un aprendizaje, a través de la comprensión de los contenidos o temas abordados.

Ahora bien para señalar los aspectos resaltantes de esta teoría considerando la modalidad de estudio de los programas universitarios, es fundamental que los profesores apliquen mecanismos de conexión así mismo 
empleen dispositivos que les permita que los estudiantes amplíen sus conocimiento a través de una fase de construcción, es conveniente resaltar la importancia del uso de las plataforma como Moodle, este es un recurso fundamental dentro de las TIC, debido a que este es un sistema completo en donde se puede articular la presencialidad con la virtualidad y obtener grandes beneficio por medio de la información que se encuentra en la medida que se comparten los conocimientos. Por lo tanto, es un medio que se articula en función de que los estudiantes logren alcanzar los objetivos planteados.

Por último, se proponer desde una concepción amplia de acuerdo con los autores estudiados en este documento un modelo de evaluación innovadora con aplicación en las TIC. Se distinguen cuatros procedimientos:

1. Procedimiento Automático: se trata de conectarse con un ordenador para ser evaluado, en algunos casos las respuestas son emitida de manera rápida en otros casos se debe esperar, depende de las estrategias, técnicas e instrumentos empleados.

2. Procedimiento Técnico: está relacionado con las tareas, se pueden clasificar en trabajos, ensayos, pruebas tipo test, foros, debates, grupo de discusión entre otros y están enfocadas en evaluar tanto el producto como el proceso, están dirigidas a desarrollar competencias, alcanzar metas, lograr objetivos. Es importante considerar los recursos y su funcionamiento (Plataforma).

3. Procedimiento Actitudinal: es donde se consideran las necesidades de fomentar la cooperación, comunicación, negociación en los estudiantes, para que exista una interacción acorde entre moderador y estudiante, así como entre los estudiantes y los recursos de igual manera entre los mismos estudiantes.

4. Procedimiento Formativo: es el que se realiza con el apoyo de la tecnología para lo cual es importante la planificación de tareas. De igual manera se trata de que los estudiantes conozcan que competencias 
han logrado de lo contrario tener información para mejorar su aprendizaje.

\section{Conclusión}

Con respecto a este tema existen muchas investigaciones por lo tanto muchos logros alcanzados, aunque, se evidencia un vacío el cual pudiera estar relacionado, con la aplicación de técnicas e instrumentos, también con las estrategias y funciones de la evaluación. Parece estar clara cuales son los objetivos de la evaluación (para qué y qué evaluar), pero, su aplicabilidad en algunos contextos presenta deficiencia: ¿será por el desconocimiento de los docentes al respecto?; ¿Le restan importancia al procedimiento?; ¿Se están enfocando realmente en el aprendizaje de los estudiantes?.

Ahora bien para esbozar la importancia de las plataformas virtuales en los tiempos actuales, este es un proceso que requiere de una cultura que se debe fortalecer desde los centros universitarios, seleccionando docentes con un perfil amplio en la rama tecnológica que maneje de forma adecuada este tipo de recursos, la mayoría de los profesores solo conoce lo básico de las TIC, por tal motivo son profesores tradicionalistas que no asumen los retos de lo nuevo de innovar a través de nuevas vivencias.

Aprender a distancia es un recorrido que apenas está iniciando en algunas universidades, porque algunos profesores tradicionalistas temen al cambio, también se aprecia que los profesores deben trabajar más con los recursos tecnológicos, por otro lado, es una experiencia gratificante, es una alternativa en tiempo de contingencia, una emergencia en la que se está sumergido ahora con la pandemia del COVID-19. Cabe destacar que la plataforma Moodle es un servicio interesante que atrae la atención de muchas personas, pero no todos tienen la habilidad para seguir las actividades o seguir instrucciones en este sentido, por eso sería propicio que desde el contexto universitario se empleen espacios con profesores que orienten para manejar 
este tipo de recursos.

Verdaderamente se ha avanzado mucho con la valoración del aprendizaje, en tal sentido hay muchos logros a favor, en cuanto a: las técnicas de evaluación y los instrumentos, aprender en base a las competencias requeridas en cada programa, estrategia para evaluar, evaluación del aprendizaje en atención a las TIC, principios y propósitos de la evaluación. Estos avances señalan que la evaluación es un proceso que implica lograr metas, alcanzar objetivos en función de que el estudiante adquiere las competencias que le permita desarrollar capacidades para mejorar el aprendizaje. También que la evaluación de los aprendizajes permite conectar lo teórico con la práctica a través de un proceso formativo basado en una evaluación formativa de retroalimentación.

\section{Referencias}

Barberà, E. (2016). Aportaciones de la tecnología a la e-Evaluación. RED: Revista de Educación a Distancia, 50(4), 1-10, e-ISSN: 1578-7680. Recuperado de: https://digitum.um.es/digitum/handle/10201/99853

Barbera, N., \& Inciarte, A. (2012). Fenomenología y hermenéutica: dos perspectivas para estudiar las ciencias sociales y humanas. Multiciencias, 12(2), 199-205, e-ISSN: 1317-2255. Recuperado de: https://www.redalyc. org/articulo.oa?id=90424216010

Bautista, N. (2016). Problemas Éticos y Representaciones Sociales de la Evaluación en la Universidad. Revista Iberoamericana de Evaluación Educativa, 9(1), 127-144, e-ISSN: 1989-0397. Recuperado de: https://doi.org/10.15366/riee2016.9.1.008

Heidegger, M. (1989). Contribuciones a la filosofía (Del acontecimiento).

Introducción, traducción y notas de Pablo Oyarzun R. Santiago, Chile: Editorial Contenido.

López, V., \& Palacios, A. (2012). Percepción de los futuros docentes sobre 
los sistemas de evaluación de sus aprendizajes. Teoría de la Educación. Educación y Cultura en la Sociedad de la Información, 13(3), 317-341, e-ISSN: 1138-9737. Recuperado de:

https://www.redalyc.org/articulo.oa?id=201024652015

Mager, R. (1975). Medición del intento educativo. Buenos Aires, Argentina: Ediciones Guadalupe.

Monje, C. (2011a,b). Metodología de la Investigación Cuantitativa y Cualitativa: Guía Didáctica. Trabajo de Grado. Neiva, Colombia: Universidad Surcolombiana.

Peñalosa, E. (2010). Evaluación de los aprendizajes y estudio de la interactividad en entornos en línea: Un modelo para la investigación. RIED: Revista Iberoamericana de Educación a Distancia, 13(1), 17-38, e-ISSN: 1138-2783. Recuperado de: https://www.redalyc.org/articulo.oa?id=331427212002

Pérez, M., Enrique, J., Carbó, J., \& González, M. (2017). La evaluación formativa en el proceso enseñanza aprendizaje. Edumecentro, 9(3), 263-283, e-ISSN: 2077-2874. Recuperado de:

http://scielo.sld.cu/scielo.php?script=sci arttext\&pid=S2077$\underline{28742017000300017}$

Púñez, F. (2015). Evaluación para el aprendizaje: una propuesta para una cultura evaluativa. Horizonte de la Ciencia, 5(8), 87-97, e-ISSN: 23044330. Recuperado de:

https://revistas.uncp.edu.pe/index.php/horizontedelaciencia/article/view $\underline{1295}$

Rosales, M. (2014). Proceso evaluativo: evaluación sumativa, evaluación formativa y Assesment su impacto en la educación actual. En Congreso Iberoamericano de Ciencia, Tecnología, Innovación y Educación. (págs. 1-13). Artículo 662, ISBN: 978-84-7666-210-6. Buenos Aires, Argentina: Organización de Estados Iberoamericanos 
para la Educación, la Ciencia y la Cultura.

Ruiz, C. (2001). La evaluación de programas de formación de formadores en el contexto de la formación en y para la empresa. ISBN: 8469980726. España: Universitat Autònoma de Barcelona. Recuperado de: http://hdl.handle.net/10803/5003

Siemens, G. (2005a,b). Connectivism: A Learning Theory for the Digital Age. Journal of Instructional Technology and Distance Learning, 2(1), 3-10, e-ISSN: 1550-6908. United States: DonEI Learning, Inc.

Villamizar, J. (2005). Los procesos en la evaluación educativa. Educere, 9(31), 541-544, e-ISSN: 1316-4910. Recuperado de:

https://www.redalyc.org/articulo.oa?id=35603115 


\section{Elsy Jackelin Bracho Hernández \\ e-mail: docelsybracho@gmail.com}

Nacida en Puerto Cabello, estado Carabobo,

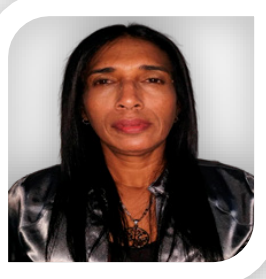
Venezuela, el 25 de mayo del año 1970. Licda. en Educación, egresada en el año 2008 por la Universidad Bolivariana de Venezuela (UBV); Magister en Educación Matemática en el 2015 egresada de la Universidad de Carabobo (UC); Profesora a nivel de primaria, secundaria, desde el año 2007; Profesora de pregrado desde el año 2009 hasta el 2014 en la Universidad Bolivariana de Venezuela, Eje Central Municipalizada y postgrado por la misma universidad en la Maestría de Educación desde el año 2017 hasta la actualidad; Profesora del programa especialización en Dirección y Supervisión Educativa; coordinadora por dos años de la Universidad Nacional Experimental Francisco de Miranda (UNEFM); actualmente soy docente titular y directora de la Escuela Primaria Bolivariana Próspero Agustín Ocando. 